\title{
ON THE AXIAL FORCE IDENTIFICATION IN EULER-BERNOULLI BEAMS WITH UNKNOWN BOUNDARY CONDITIONS
}

\author{
Margaux Geuzaine ${ }^{1}{ }^{2}$, Francesco Foti $^{13}$, and Vincent Denoël ${ }^{1}$ \\ ${ }^{1}$ Structural \& Stochastic Dynamics, University of Liège, Belgium \\ Allée de la Découverte 1, 4000 Liège, Belgium \\ e-mail: mgeuzaine@uliege.be, francesco.foti@polimi.it,v.denoel@uliege.be \\ ${ }^{2}$ F. R. S.-FNRS, National Fund for Scientific Research, Belgium \\ ${ }^{3}$ Department of Civil and Environmental Engineering, Politecnico di Milano, Italy
}

Keywords: Axial-force identification, Health-monitoring, Safety assessment, Rotational stiffness, Differential Evolution.

\begin{abstract}
Identification of the tensile force in slender axially-loaded structural elements, such as stay cables, metallic bars and tie-rods, is of paramount importance for health monitoring and safety assessment purposes. Dynamic testing techniques provide the ground for quick and cheap identification strategies, based on the knowledge of: (a) a set of identified natural frequencies, and (b) a structural model that relates natural frequencies to the axial-force value. Within this framework, reliability of the identified axial force values depends, among other factors, on the predictive capabilities of the underlying structural model. Errors may arise, in particular, from the modeling of boundary conditions. The present paper analytically investigates the effect of boundary conditions on the modal properties of axially loaded Euler-Bernoulli beam elements. Starting from theoretical results obtained on this archetypal structural model, a numerical procedure for the identification of the axial force in beam elements with unknown bending stiffness and boundary conditions is then presented. Parametric analyses are carried out to quantitatively assess the accuracy of the proposed identification algorithm in the case of frequencies contaminated by measurement errors.
\end{abstract}




\section{INTRODUCTION}

Dynamic testing techniques are widely used to get estimates of the modal properties of slender axially-loaded one-dimensional structural elements, such as stay cables, diagonal braces, truss elements and tie-rods of vaulted historical masonry structures. Knowledge of a set of measured natural frequencies of the beam, along with a suitable structural model updating strategy, then, can be used to identify the value of the axial force acting on the element, which is of paramount importance for structural health monitoring and safety assessment purposes. Within this framework, reliability of the identified axial force values depends, among the other factors, on the predictive capabilities of the underlying structural model.

Frequency-based axial force identification procedures often rely on the assumption of ideal boundary conditions, in the form of either perfectly hinged or perfectly clamped end sections (see e.g. [1, 7]). A more realistic structural scheme could be defined, however, by considering equivalent translational and rotational springs at the beam end sections to model the flexibility of the restraint devices and of the support structures (e.g. deck and tower for cables in stayed bridges). Proper definition of equivalent springs strongly depends on the particular technology adopted to realize the restraints and is inherently related to several different sources of uncertainties, such as those related to geometric imperfections and aging of the support devices.

The present paper presents an analytical investigation of the effects of the boundary conditions on the modal properties of axially-loaded Euler-Bernoulli beam elements whose end sections are restrained by rotational springs. Starting from theoretical results obtained on this archetypal structural model, a numerical procedure for the identification of the axial force in beam elements with unknown bending stiffness and boundary conditions is then presented. Parametric analyses are carried out to quantitatively assess the accuracy of the proposed identification algorithm in the case of frequencies contaminated by measurement errors.

\section{FORMULATION OF THE PROBLEM}

\subsection{Free-vibrations of the Euler-Bernoulli beam model}

Let us consider a straight axially-loaded one-dimensional structural element, with constant bending stiffness $(E I)$ and mass per unit of length $(m)$. Transverse displacements of the centerline are described through the function $v=v(x, t)$, where $x \in[0, l]$ is a coordinate spanning the free length $(l)$ of the element and $t$ is the time. By neglecting sag-extensibility and shear deformability effects, undamped planar transverse vibrations are governed by the partial differential equation (see e.g. [8]):

$$
E I \partial_{x}^{4} v-T \partial_{x}^{2} v+m \partial_{t}^{2} v=0
$$

where $T>0$ is the axial force (see Fig. 1).

By introducing the characteristic frequency $\omega_{0}$ and the non-dimensional bending stiffness $\varepsilon$ :

$$
\omega_{0}=\sqrt{\frac{T}{m l^{2}}}, \varepsilon=\sqrt{\frac{E I}{T l^{2}}},
$$


the Eq. (1) can be re-stated in the following non-dimensional form:

$$
\varepsilon^{2} \partial_{\xi}^{4} \bar{v}-\partial_{\xi}^{2} \bar{v}+\partial_{\tau}^{2} \bar{v}=0
$$

where $\xi=\frac{x}{l} \in[0,1], \tau=\omega_{0} t$ and $\bar{v}(\xi, \tau)=\frac{v(x(\xi), t(\tau))}{l}$.

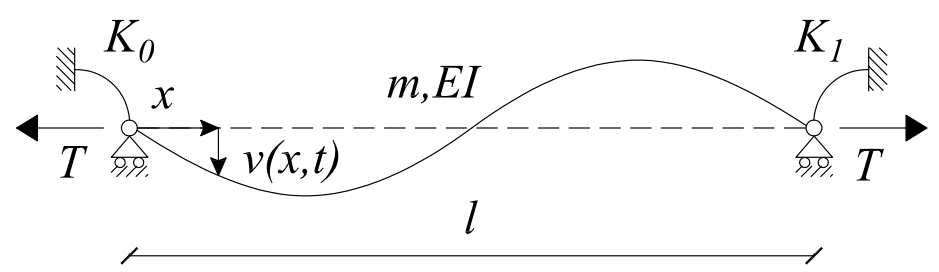

Figure 1: Schematic representation of a straight one-dimensional structural element subject to a tensile load $T$.

Values of $\varepsilon$ typical of slender structural elements, such as stay cables and masts, are lower than 2\% (see e.g. [1, 7, 15]). Thicker structural elements such as diagonal braces, truss elements, short cables and tie-rods of vaulted historical masonry structures, instead, can be characterized by values of non-dimensional bending stiffness in the broader range: $0.02 \lesssim \varepsilon \lesssim 1$ (see e.g. [11, 12, 13, 17]). It's worth noting that the governing equation (3) is singularly perturbed for small values of $\varepsilon$, typical of slender elements, hinting the existence of boundary layers in the mode shapes and possible ill-conditioning of numerical solvers (see e.g. [10, 5]).

General solutions of Eq. (3) can be expressed as $\bar{v}(\xi, \tau)=\phi(\xi) \sin (\bar{\omega} \tau-\theta)$, where $\bar{\omega}$ is a non-dimensional vibration frequency, $\theta$ is a phase angle depending on initial conditions and $\phi(\xi)$ is a mode shape function. The vibration frequencies $\bar{\omega}$ and shape functions $\phi(\xi)$ are the solutions of a fourth order boundary value problem defined by the ordinary differential equation:

$$
\varepsilon^{2} \phi^{I V}-\phi^{I I}-\omega^{2} \phi=0, \xi \in[0,1]
$$

along with suitable conditions modeling the cable restraints.

Ideal boundary conditions are often introduced, in the form of either perfectly hinged or perfectly clamped cable end sections. In many practical circumstances, however, this modeling strategy can lead to an oversimplified representation of the structural dynamics, naturally claiming for the adoption of elastically supported beam models (see e.g. [14, 16, 2]). In the present work, two rotational springs with stiffness $K_{i}(i=1,2)$ are assumed to be attached at the end sections of the element (see Fig. 1). The degree of fixity of the rotational restraints is then defined by introducing the non-dimensional parameters:

$$
\rho_{i}=\frac{K_{i}}{K_{i}+\varepsilon T l}, i=0,1
$$

It is worth noting that the parameter $\rho_{i}$ can take values in the unit interval $[0,1]$, being strictly equal to zero for $K_{i}=0$, i.e. for a perfectly hinged end section. The upper bound 
limit case $\rho_{i}=1$, instead, is asymptotically approached as $K_{i} \rightarrow \infty$, i.e. for a perfectly clamped end section.

By accounting for the definitions in Eq. (5), the boundary conditions associated to Eq. (4) read:

$$
\left\{\begin{array}{l}
\phi(0)=0 \\
\phi(1)=0 \\
\left(1-\rho_{0}\right) \varepsilon^{2} \phi^{\prime \prime}(0)-\rho_{0} \varepsilon \phi^{\prime}(0)=0 \\
\left(1-\rho_{1}\right) \varepsilon^{2} \phi^{\prime \prime}(1)+\rho_{1} \varepsilon \phi^{\prime}(1)=0
\end{array}\right.
$$

The general solution of Eq. (4) can be expressed as a combination of trigonometric and exponential functions:

$$
\phi\left(\xi ; z_{1}, z_{2}\right)=a_{1} \sin \left(z_{1} \xi\right)+a_{2} \cos \left(z_{1} \xi\right)+a_{3} \exp \left(-z_{2} \xi\right)+a_{4} \exp \left(-z_{2}(1-\xi)\right)
$$

where $a_{i}(i=1, \ldots, 4)$ are real integration constants and the arguments $z_{k}(k=1,2)$ are defined as:

$$
z_{i}(\bar{\omega} ; \varepsilon)=\frac{1}{\varepsilon \sqrt{2}} \sqrt{(-1)^{i}+\sqrt{1+4 \varepsilon^{2} \bar{\omega}^{2}}}, i=1,2
$$

Notice that the use of exponential instead of hyperbolic functions in Eq. (7) is more appropriate to highlight the existence of two boundary layers when $\varepsilon<<1$.

Substitution of Eqs. (7) and (8) in (6) yields the algebraic eigenvalue problem:

$$
\mathbf{B}\left(\bar{\omega} ; \varepsilon, \rho_{0}, \rho_{1}\right) \mathbf{a}=\mathbf{0}
$$

where $\mathbf{a}=\left(A_{1}, \ldots, A_{4}\right)^{T}, \mathbf{0}$ is a null column matrix of size $4 \times 1$, and $\mathbf{B}$ is a $4 \times 4$ boundary condition matrix whose entries depend on both the non-dimensional bending stiffness $\varepsilon$ and the degree of fixity parameters $\rho_{i}(i=0,1)$.

The non-dimensional natural frequencies $\bar{\omega}_{k}\left(k \in \mathbb{N}^{+}\right)$coincide with the roots of the characteristic equation $\operatorname{det}\left(\mathbf{B}\left(\bar{\omega} ; \varepsilon, \rho_{0}, \rho_{1}\right)\right)=0$. Generally speaking, they will be a function of the non-dimensional bending stiffness $\varepsilon$ and of the two variables $\rho_{0}$ and $\rho_{1}$, i.e. $\bar{\omega}_{k}=\bar{\omega}_{k}\left(\varepsilon, \rho_{0}, \rho_{1}\right), k \in \mathbb{N}^{+}$. Symmetry, however, allows to conclude that the nondimensional natural frequencies $\bar{\omega}_{k}$ should depend on a symmetric transformation of the variables $\rho_{0}$ and $\rho_{1}$ that can be arbitrarily, but conveniently, defined as:

$$
p=\frac{1}{2}\left(\rho_{0}+\rho_{1}\right), 0 \leq p \leq 1
$$

Dependency of the non-dimensional natural frequencies on $p$, then, can be fully highlighted by writing: $\bar{\omega}_{k}=\bar{\omega}_{k}(\varepsilon, p), k \in \mathbb{N}^{+}$. It's worth noting that the ideal limit cases of doubly-hinged and doubly-clamped elements correspond, respectively, to set $p=0$ and $p=1$.

Exact closed-form solutions of the characteristic equation are only available for the doubly-hinged case (see e.g. [8]). Asymptotic closed-form solutions can also be derived, for small values of the non-dimensional bending stiffness parameters $\varepsilon$ typical e.g. of stay cables. By extending the results firstly presented in [6] to deal with a beam element 
supported by rotational springs at both end sections, indeed, the following result can be obtained:

$$
\bar{\omega}_{k}=k \pi\left[1+2 p \varepsilon+\left(\frac{(k \pi)^{2}}{2}+4 p^{2}\right) \varepsilon^{2}\right], k \in \mathbb{N}^{+}+o\left(\varepsilon^{2}\right)
$$

For arbitrary boundary conditions and non-dimensional bending stiffness values, the characteristic equation can be numerically solved e.g. through a sequential application of a root finding algorithm based on the bisection method. Within this context, it is worth noting that, thanks to the scaling of the governing equations adopted in the present work, the determinant of the boundary condition matrix $\mathbf{B}$ is bounded and with leading order terms of order one for vanishingly small values of $\varepsilon$. This makes simple numerical root finding algorithms well suited to find the natural frequencies of the system for over a wide interval of non-dimensional bending stiffness values, ranging from elements characterized by values of $\varepsilon$ in the order of $0.5-1$ to very slender ones, with $\varepsilon$ in the order of few percents or lower.

\subsection{Parameter identification algorithm}

Once the eigenvalues $\bar{\omega}_{k}=\bar{\omega}_{k}(\varepsilon, p)$ are known through an application of the semianalytic model described in Section (2.1), multiplication by the characteristic frequency $\omega_{0}=\omega_{0}(T, m, l)$ gives the natural frequencies:

$$
\omega_{k}=\omega_{0}(T, m, l) \cdot \bar{\omega}_{k}(\varepsilon, p), k \in \mathbb{N}^{+}
$$

Standard vibration testing techniques (see e.g. [20, 9]) can be applied to obtain the first $M$ natural frequencies of the beam: $\omega_{k}^{*}(k=1, \ldots, M)$. The difference between calculated and measured natural frequencies, then, can be quantitatively assessed by introducing the cost (or objective) function:

$$
F_{o b j}=\sqrt{\sum_{k=1}^{M}\left(1-\frac{\omega_{k}}{\omega_{k}^{*}}\right)^{2}}
$$

The unknown model parameters $\boldsymbol{X}=\left(\omega_{0}, \varepsilon, p\right)^{T} \in \mathbb{R}^{3}$ can be identified by solving the non-linear optimization problem:

$$
\text { Find } \boldsymbol{X}_{o p t} \text { s.t. } F_{o b j}\left(\boldsymbol{X}_{o p t}\right)=\inf \{(\boldsymbol{X})\}
$$

with the physical constraints: $\omega_{0}>0, \varepsilon>0,0 \leq p \leq 1$. Once the optimal parameters $\omega_{0}, \varepsilon$ and $p$ are known from the solution of (14), by assuming that the length $(l)$ and the mass per unit of length $(m)$ are known without uncertainties, the axial-force $T$ can be easily calculated (see Eq. (2)) as $T=m l^{2} \omega_{0}^{2}$.

The non-linear optimization problem defined in Eq. (14) is characterized by several characteristic features that should guide the selection of an appropriate solution algorithm. Due to unavoidable measurement errors affecting the natural frequencies $\omega_{k}^{*}(k=1, \ldots, M)$, the landscape of the cost function $(12)$ is characterized, in general, by many local minima. Moreover, the sensitivity of cost function (12) with respect to the parameter $p$ tends to be substantially negligible for small values of the non-dimensional bending stiffness $\varepsilon$ typical of slender elements, as it can be easily inferred by inspecting the asymptotic solution of the eigenvalue problem (Eq. (11)). 
Gradient-based optimization algorithms, hence, are not well suited for the particular problem at hand, since they are prone to get trapped in local minima and their iteration operators could potentially be ill-conditioned for small values of $\varepsilon$. Within the class of gradient-free algorithms, the family of Differential Evolution algorithms, firstly proposed by Storn and Price [18, 19], has shown excellent performances in finding the global optimum of non-linear, non-convex, multi-modal and non-differentiable functions (see e.g. the review in [3]). DE is an Evolutionary Algorithm (EA) that iteratively operates on a population of candidate solutions made of $N P$ parameter vectors. The initial population is randomly chosen and offsprings are generated by perturbing trial solutions with scaled differences of randomly selected population elements. As the number of iterations grows, the characteristic size of these differences tend to automatically adapt to the natural scales of the objective landscape [3]. It's worth noting that the peculiar strategy adopted to generate offsprings and evolve the population of candidate solution makes DE algorithms able to deal with objective functions characterized by low or moderately low sensitivity with respect to one ore more variables of the searching space without numerical problems. Selection of the better fitted elements of the population is performed through a one-to-one parent/offspring competition scheme. The physical constraint are enforced through a simple penalty criterion and the iterations are performed until a termination criterion is satisfied.

In the present work, a custom implementation of a well-known variant of the DE algorithm proposed by [4] has been adopted to solve the non-linear optimization problem in Eq. (14). The termination criterion has been defined such that iterations are stopped whenever one of the following conditions is satisfied: (a) the relative difference between

the best and worst objective function values $\Delta=\frac{F_{o b j}^{w o r s t}-F_{o b j}^{b e s t}}{\max \left\{F_{o b j}^{w o r s t}, 1\right\}}$ of a population is below a given threshold Toll (cf. the Diff termination criterion in [21]), (b) the value of the cost function is lower than a prescribed value $V$, (c) the number of iterations $N I T$ is equal to the maximum number of iterations $M A X I T$.

\section{APPLICATIONS}

The performances of the proposed parameter identification strategy have been assessed through extensive numerical testing. Results will be presented in the following for an axially-loaded beam element characterized by $\omega_{0}=5.66 \mathrm{rad} / \mathrm{s}$ and $T=4000 \mathrm{kN}$. Three different values of non-dimensional bending stiffness $(\varepsilon=0.02,0.1,0.5)$ have been considered, along with five different boundary conditions: (I) $p=0$ (doubly-hinged beam), (II) $p=0.25$, (III) $p=0.50$, (IV) $p=0.75$ and (V) $p=1$ (doubly-clamped beam).

In order to simulate experimental input data, the eigenvalue problem $(9)$ has been numerically solved to get the first five natural frequencies. These frequencies, then, have been corrupted by multiplying the nominal values by a unit-mean and low intensity Gaussian noise, to account for the effects of measurement errors. Different values of noise intensity, ranging from 0 to $2.5 \%$, have been considered. For each noise intensity value, a sample of 10 sets of noisy natural frequencies has been randomly generated. The optimization problem (14) has been solved for each set of numerically generated input natural frequencies by running the DE algorithm ("DEGL/SAW/bin" scheme, with scale factor $F=0.8$ and crossover parameter $C R=0.9$, see [4] for further details), starting from a population of $N P=30$ trial solutions randomly chosen in the initial search space $P \subset \mathbb{R}^{3}: 10^{-5} \leq \omega_{0} \leq 50,10^{-5} \leq \varepsilon \leq 5,0 \leq p \leq 1$. The parameters of the termination 
criterion have been set up as: $V=10^{-6}$, Toll $=10^{-7}$ and $M A X I T=1000$.

Figures (2)-(4) show the results of the proposed identification algorithm as a function of the noise intensity. The results are averaged over the number of runs $N R$ of the algorithm for each different noise intensity level (i.e. $N R=10$ ) and expressed in terms of: (a)-(c) the identified values of the parameters $\omega_{0, \text { ave }}, \varepsilon_{\text {ave }}$ and $p_{\text {ave }} ;(\mathrm{d})$ the relative error on the element axial force, $\Delta T=\frac{T_{a v e}-T^{*}}{T^{*}}$, where $T_{\text {ave }}$ is the average identified value of the axial force and $T^{*}$ is the target value (i.e. $T^{*}=4000 \mathrm{kN}$ ); (e) is the average value of the cost function associated to the identified optimal set of parameters (denoted as $F_{o b j}^{b e s t}$ in the figures); (f) is the average number of iterations of the DE algorithm.

Figure 2 shows the results for a beam element with target bending stiffness value $\varepsilon=0.02$ typical of slender elements, such as stay cables. The identification strategy gives fairly accurate results in terms of parameters $\omega_{0}$ and $\varepsilon$ for all values of noise intensity herein considered. As expected from the inspection of the asymptotic solution of the eigenvalue problem (Eq. (11)), the boundary conditions do not affect significantly the cost function of the optimization problem and, as a consequence, the identification algorithm is not able to correctly identifying the parameter $p$. As already noticed by the authors in [6], the identification procedure tends, for each value of noise intensity, to a mean value of $p$ equal to about 0.5 . This latter value coincides with the mean value of $p$ within the randomly generated population members of the DE algorithm. On the overall, in spite of an highly inaccurate identification of the degree of fixity of the beam end sections, the procedure gives a good estimate of the axial force. Errors on the axial force obviously increase at the increase of the noise intensity and are higher for the two boundary conditions corresponding to the limit cases of doubly-hinged (label I, $p=0$ ) and doubly-clamped (label V, $p=1$ ) beams. Figure 2(e) allows to appreciate how measurements errors, herein associated to non-zero noise intensity values, determines a significant jump in the estimated minimum values of the cost function. Figure 2(f) allows to appreciate how for non-zero noise-intensity values iterations are mostly terminated because the maximum number of iterations (MAXIT) is reached. This is mainly due to the already discussed lacking of convergence on the degree of restraint parameter $p$ (see also [6]).

Figure 3 shows the results for a beam element with target bending stiffness value $\varepsilon=0.1$. The identification algorithm gives very accurate predictions of all parameters $\omega_{0}, \varepsilon$ and $p$ for small values of the noise intensity (i.e. lower than $0.5 \%$ ). By increasing the noise intensity, the accuracy in the predictions of the degree of restraint parameters $p$ rapidly deteriorates, while the errors on both $\omega_{0}$ and $\varepsilon$ remain acceptable with maximum discrepancies from the target values in the order of $\pm 15 \%$, depending on the boundary conditions. The maximum relative errors on the identified axial force are in the order of $\pm 15 \%$ for small values of the noise intensity (i.e. lower than $0.5 \%$ ), but rapidly increase up to about $\pm 25 \%$ for a noise intensity value equal to $2.5 \%$. Figures $3(\mathrm{a})$ and $3(\mathrm{~d})$ also allows one to appreciate how, for the non-dimensional bending stiffness value herein considered, the proposed identification algorithm tends to systematically underestimate the axial force for the boundary conditions labeled (I) and (II), i.e. for $p$ respectively equal to 0 (doublyhinged beam) and 0.25 ; on the other hand, the axial force is systematically overestimated for the boundary conditions labeled (IV) and (V), i.e. for $p$ respectively equal to 0.75 and 1 (doubly-clamped beam). The opposite trend is remarked for the non-dimensional bending stiffness $\varepsilon$ (see Fig. 3(b)). These trends can be easily explained, through an inspection of Fig. 3(d), by noticing that the algorithm tends to: (a) overestimate the degree of fixity of the restraints in cases (I) and (II); and (b) underestimate the degree of 
fixity of the restraints in cases (I) and (II).

Figure 4 shows the results for a beam element with target bending stiffness value $\varepsilon=0.5$, that can be considered as representative e.g. of diagonal braces, truss elements and tie-rods. Differently than in the previous cases, the boundary conditions are identified with good accuracy for all values of noise intensity herein considered, except that for the case labeled as (I), corresponding to $p=0$ (doubly-hinged beam), which is associated to errors on $p$ in the order of $25 \%$. More in details, the accuracy of the identified values of $p$ decreases with the increasing of the flexibility of the restraints. The identified values of the parameters $\omega_{0}$ and $\varepsilon$, on the other hand, are affected in general by significant discrepancies with respect to the target values. As a consequence, also the errors affecting the identified axial force are relevant. More in details, it is worth noting that errors on the non-dimensional bending stiffness parameter $\varepsilon$ reach the $60 \%$, depending on the boundary conditions and on the noise intensity value. Although difficult to evaluate in slender structural elements such as stay cables (associated to small values of $\varepsilon$ ), the bending stiffness of thicker structural elements such as diagonal braces, truss elements and tierods can often be easily estimated on the basis of available information on the geometric and mechanical properties of the structural element. Whenever the bending stiffness is known, however, the proposed algorithm can be easily re-formulated by assuming that $\omega_{0}$ and $p$ are the only unknown parameters, leading to a more precise evaluation of the beam axial force.

\section{CONCLUSIONS}

An analytical model for transverse vibrations of an axially loaded Euler-Bernoulli beam with partially restrained end conditions has been presented and used as the basis to develop a numerical parameter identification strategy. Parametric analyses have been carried out to quantitatively assess the performances of the proposed identification algorithm, for a broad range of structural configurations, characterized by different values of nondimensional bending stiffness $\varepsilon$ and boundary conditions, that cover a wide spectrum of applications, such as stay cables, diagonal braces, truss elements and tie-rods of vaulted historical masonry structures. On the overall, the proposed procedure gives accurate estimates of the beam axial force for small and moderately small values of $\varepsilon$, in the order of $\varepsilon \lesssim 0.1$. For higher values of $\varepsilon$, based on the critical discussion of the results of the proposed identification strategy, a simplified algorithm relying on a preliminary estimation of the bending stiffness of the beam is envisaged to reduce errors on the identified axial force.

\section{ACKNOWLEDGMENTS}

The project is funded by the Wallonia Public Service. It is carried out by the University of Liège and the company V2i (www.v2i.be). M.G. acknowledges the support of the FNRS (Belgian Fund for Scientific Research).

\section{REFERENCES}

[1] E. Caetano, Cable vibrations in cable-stayed bridges, I. A. for B. and S. Engineering (IABSE) Ed., 2007. 

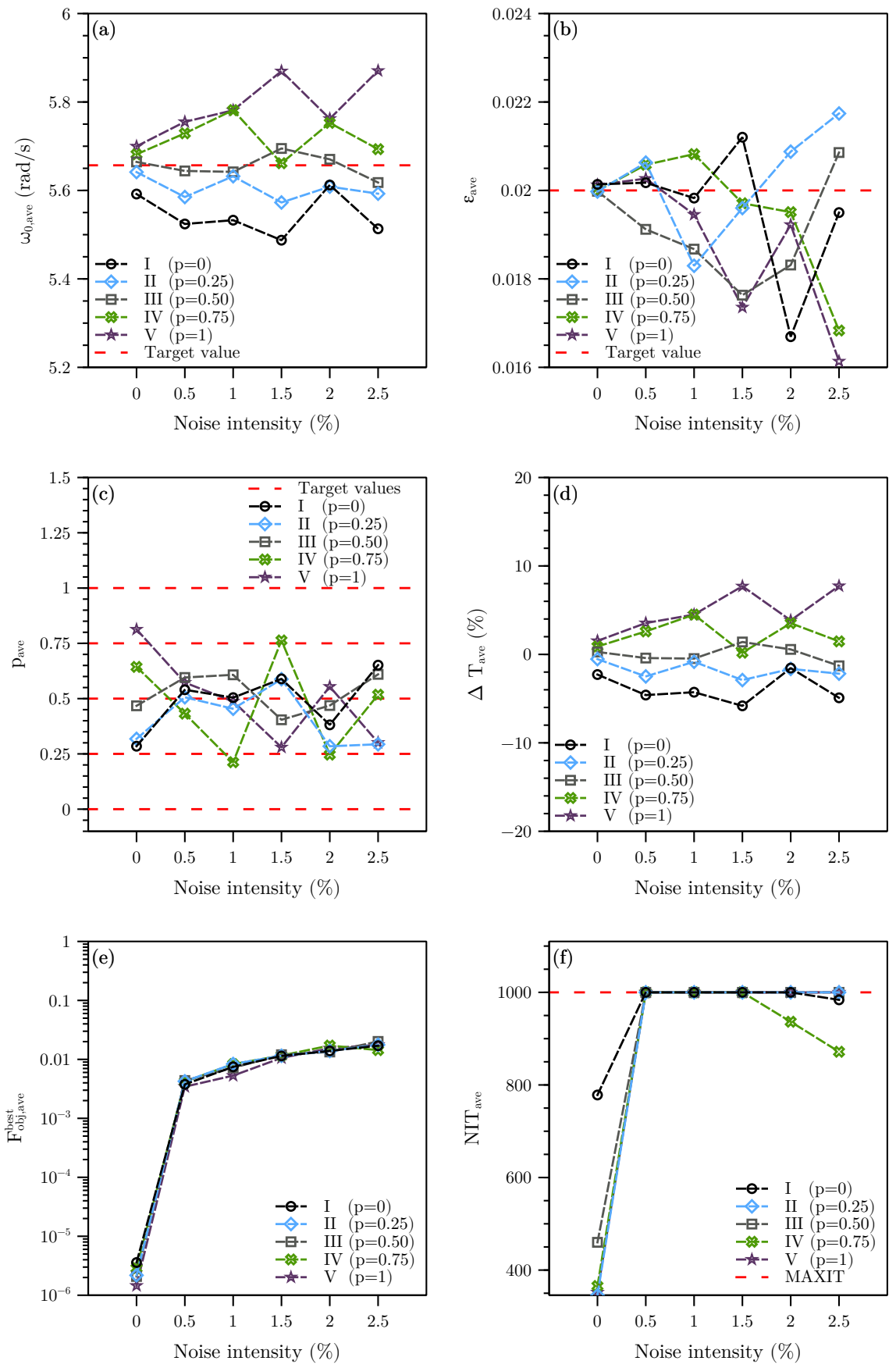

Figure 2: Results of the identification algorithm, averaged on ten runs, as a function of the noise intensity. (a) Characteristic frequency $\omega_{0}$ (target value: $\omega_{0}=5.66 \mathrm{rad} / \mathrm{s}$ ), (b) non-dimensional bending stiffness $\varepsilon$ (target value: $\varepsilon=0.02$ ), (c) degree of fixity parameter $p$, (d) relative error on the axial force $\Delta T$, (e) value of the cost function associated to the identified optimal set of parameters, $F_{o b j}^{b e s t}$ (f) number of iteration (the maximum number is $M A X I T=1000$ ). 

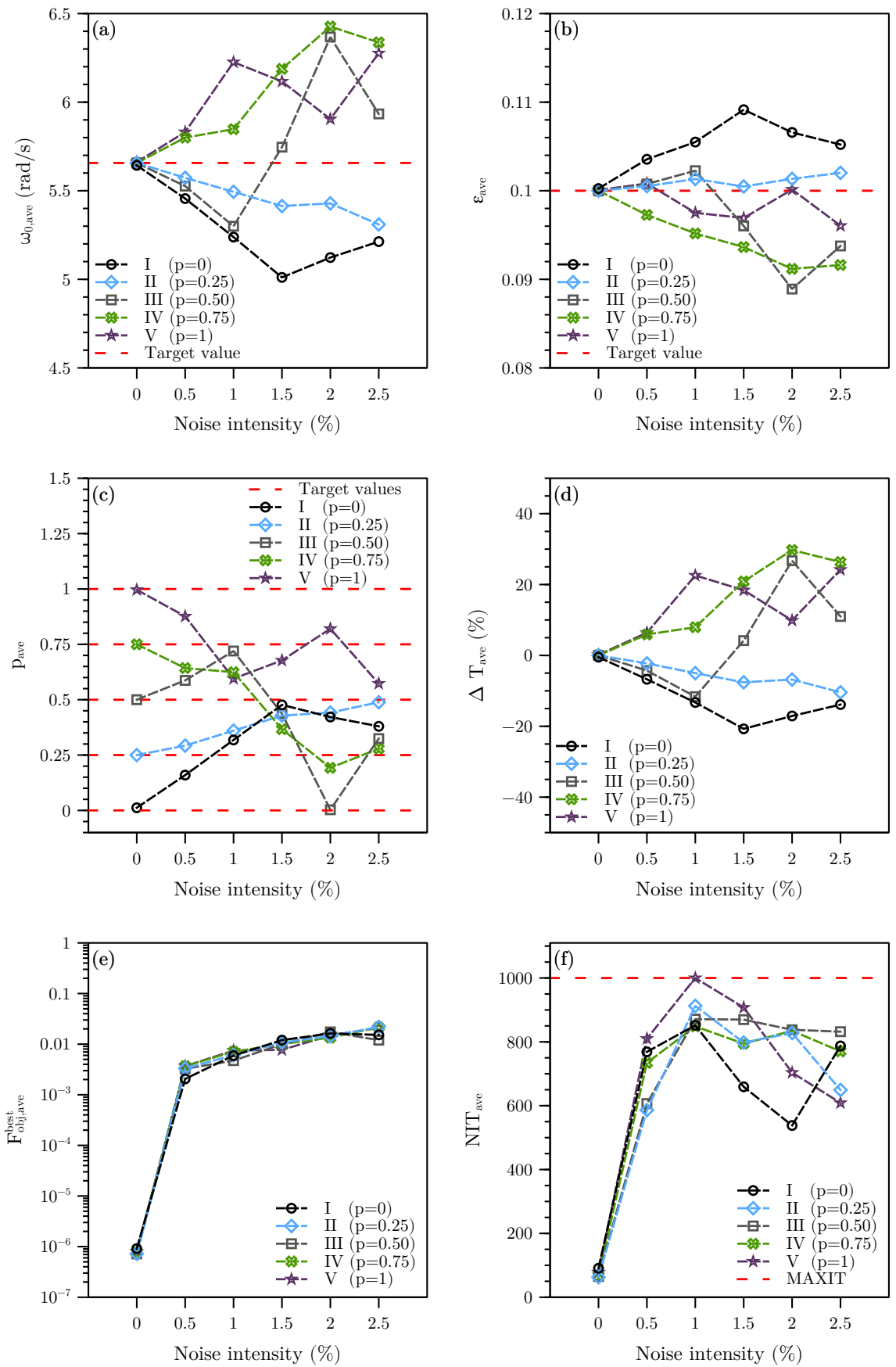

Figure 3: Results of the identification algorithm, averaged on ten runs, as a function of the noise intensity. (a) Characteristic frequency $\omega_{0}$ (target value: $\omega_{0}=5.66 \mathrm{rad} / \mathrm{s}$ ), (b) non-dimensional bending stiffness $\varepsilon$ (target value: $\varepsilon=0.1$ ), (c) degree of fixity parameter $p$, (d) relative error on the axial force $\Delta T$, (e) value of the cost function associated to the identified optimal set of parameters, $F_{o b j}^{b e s t}$ (f) number of iteration (the maximum number is $M A X I T=1000$ ). 

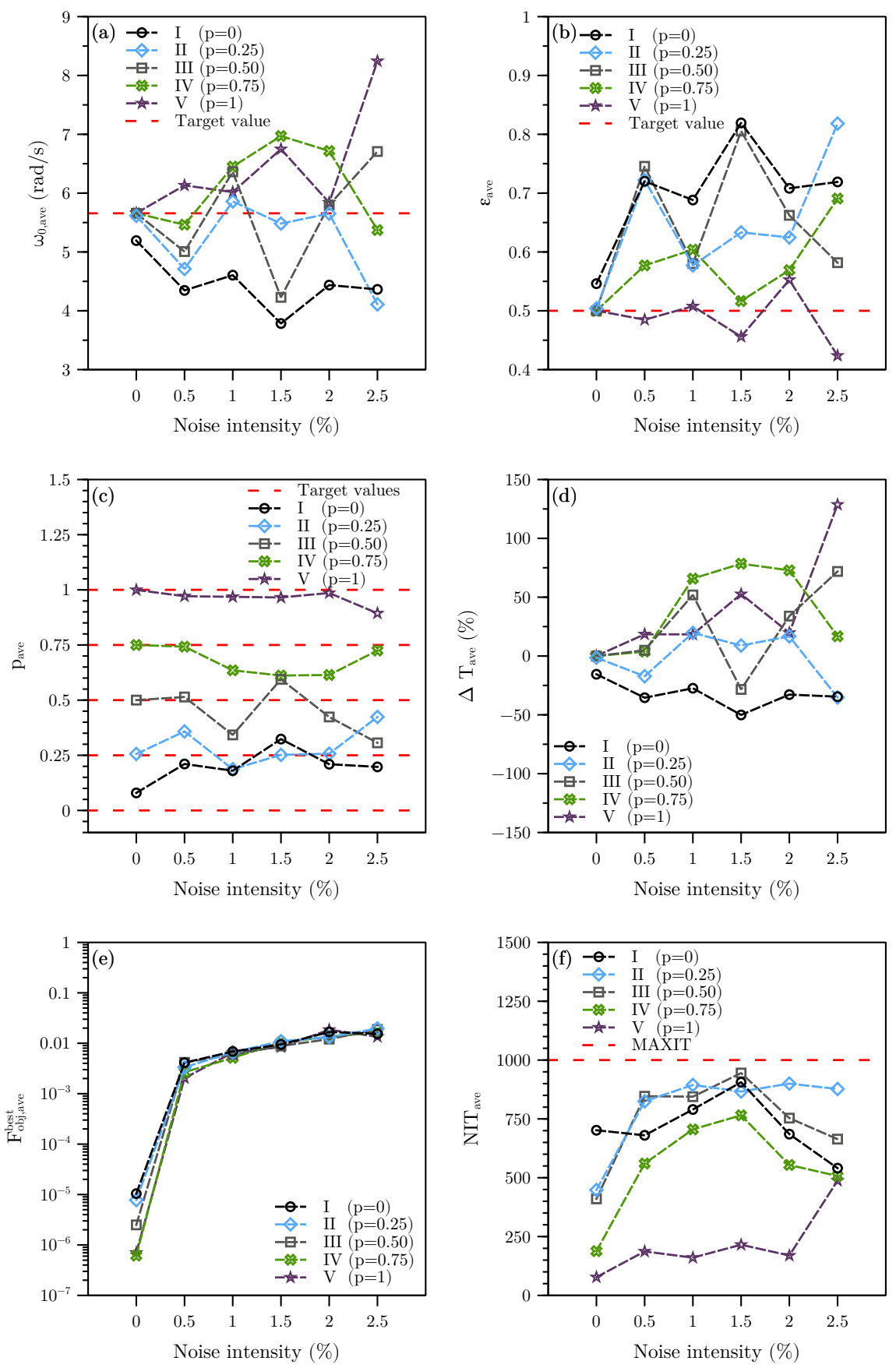

Figure 4: Results of the identification algorithm, averaged on ten runs, as a function of the noise intensity. (a) Characteristic frequency $\omega_{0}$ (target value: $\omega_{0}=5.66 \mathrm{rad} / \mathrm{s}$ ), (b) non-dimensional bending stiffness $\varepsilon$ (target value: $\varepsilon=0.5$ ), (c) degree of fixity parameter $p$, (d) relative error on the axial force $\Delta T$, (e) value of the cost function associated to the identified optimal set of parameters, $F_{o b j}^{b e s t}$ (f) number of iteration (the maximum number is $M A X I T=1000$ ). 
[2] E. Caetano, R. Bartek, F. Magalhaes, C. Keenan, G. Trippick, Assessment of cable forces at the London 2012 Olympic Stadium roof, Structural Engineering International, 4, 489-500, 2013.

[3] S. Das, S.S. Mullick, P.N. Suganthan, P.N., Recent advances in differential evolution - An updated survey, Swarm and Evolutionary Computation, 27, 1-30, 2016.

[4] S. Das, A. Abraham, U.K. Chakraborty, A. Konar, Differential evolution using a neighborhood-based mutation operator, IEEE Transactions on Evolutionary Computation, 13, 526-553, 2009.

[5] V. Denoël, E. Detournay, Multiple scales solution for a beam with a small bending stiffnes, Journal of Engineering Mechanics, 136, 69-77, 2010.

[6] F. Foti, M. Geuzaine, V. Denoël, On the identification of the axial force in stay cables with unknown boundary conditions, Proc. of the 8th UBT International Conference. on Civil Engineering, Infrastructure and Environment (IC-UBT), Pristine, Kosovo, 26th-28th October 2019.

[7] R. Geier, G.D. Roeck, R. Flesch, Accurate cable force determination using ambient vibration measurements, Structure and Infrastructure Engineering, 2, 43-52, 2006.

[8] M. Géradin, D. Rixen, Mechanical vibrations, John Wiley and Sons, Chichester (England), 1997.

[9] C.M. Harris, A.G. Piersol, Harris' shock and vibration handbook, Vol. 5. New York: McGraw-Hill, 2002.

[10] E.J. Hinch, Perturbation methods, Cambridge University Press, Cambridge, U.S., 1991.

[11] T. Kernicky, M. Whelan, E. Al-Shaer, Dynamic identification of axial force and boundary restraints in tie rods and cables with uncertainty quantification using Set Inversion Via Interval Analysis, Journal of Sound and Vibration, 423, 401-420, 2018.

[12] S. Lagomarsino, C. Calderini, The dynamical identification of the tensile force in ancient tie-rods, Engineering Structures, 27 846-856, 2005.

[13] S. Li, E. Reynders, K. Maes, G. De Roeck, Vibration-based estimation of axial force for a beam member with uncertain boundary conditions, Journal of Sound and Vibration, 332, 795-806, 2013.

[14] P. de Mars, D. Hardy, Mesure des Efforts dans les Structures a Cables, Annales TP Belgique, 6, 515-531, 1985.

[15] A.B. Mehrabi, H. Tabatabai, Unified finite difference formulation for free vibration of cables, Journal of Structural Engineering (ASCE), 124, 1313-1322, 1998.

[16] U. Pabst, P. Hagedorn, Identification of boundary conditions as a part of model correction, Journal of Sound and Vibration, 182, 565-575, 1995. 
[17] G. Rebecchi, N. Tullini, F. Laudiero, Estimate of the axial force in slender beams with unknown boundary conditions using one flexural mode shape, Journal of Sound and Vibration, 332, 4122-4135, 2013.

[18] R. Storn, K.V. Price, Differential evolution: a simple and efficient adaptive scheme for global optimization over continuous spaces, ICSI, USA, Tech. Rep. TR-95-012, 1995.

[19] R. Storn, K.V. Price, Differential evolution: a simple and efficient heuristic for global optimization over continuous spaces, Journal of Global Optimization, 11, 341-359, 1997.

[20] P. Van Overschee, B. De Moor, Subspace algorithms for the stochastic identification problem, Automatica, 29, 649-660, 1993.

[21] K. Zielinski, R. Laur, Stopping criteria for differential evolution in constrained singleobjective optimization, Advances in Differential Evolution, pp. 111-138, SpringerVerlag, Berlin, 2008. 